\title{
Prolactin-related adverse events and change in prolactin levels in pediatric patients given antipsychotics for schizophrenia and schizophrenia spectrum disorders: A systematic review
}

\author{
Eric Druyts ${ }^{1 *}$, Michael J. Zoratti ${ }^{2}$, Kabirraaj Toor ${ }^{1}$, Ping Wu${ }^{1}$, Salmaan Kanji ${ }^{3}$, Kiran Rabheru ${ }^{4}$, Edward J. Mills ${ }^{1}$
} and Kristian Thorlund ${ }^{2}$

\begin{abstract}
Background: Second-generation antipsychotics are commonly prescribed for pediatric patients with schizophrenia and schizophrenia spectrum disorders despite their lack of approval for use in children. Although considered a safer alternative to first-generation antipsychotics, there is evidence to suggest that second-generation antipsychotics may be associated with some adverse events as well as an increase in prolactin levels. The purpose of this review is to examine the risk of prolactin-related adverse events in pediatric patients using antipsychotics and to quantify changes in prolactin for this population.

Methods: Literature searches were conducted in Medline, Embase, the Cochrane Central Register of Controlled Trials, and PsycINFO databases, supplemented with review of select gray literature to identify both randomized controlled trials and observational studies on pediatric patients prescribed antipsychotic medications for schizophrenia or schizophrenia spectrum disorders. Using a narrative approach, data on adverse events were recorded and changes from baseline in prolactin were pooled, where possible, from the randomized trials. Change from baseline in prolactin was evaluated for each treatment, as well as in comparison to placebo and to other treatments. Where data was available, these changes were evaluated separately for male and female patients.

Results: Six randomized controlled trials and five observational studies, all examining the effects of second-generation antipsychotics, were selected. Literature reporting the effects of risperidone, quetiapine, aripiprazole, olanzapine, and paliperidone was identified, with varying doses. Prolactin-related adverse events were sparsely reported across studies. In evidence gathered from randomized controlled trials, risperidone, olanzapine, and two doses of paliperidone (3-5 mg/day and 6-12 mg/day) were associated with increased prolactin levels compared to baseline. With the exception of paliperidone, similar trends were observed in males and females, separately. The findings of the observational evidence served to both complement and run contrary to the randomized trials, with discrepancies attributed to differences in patient and treatment characteristics.

(Continued on next page)
\end{abstract}

\footnotetext{
*Correspondence: edruyts@gmail.com

'Faculty of Health Sciences, University of Ottawa, Ottawa, ON, Canada

Full list of author information is available at the end of the article
} 
(Continued from previous page)

Conclusions: No definitive conclusions between second-generation antipsychotic use and prolactin-related adverse events can be made based on the available literature. While some trends in prolactin level changes emerged, this was based on few trials with small sample sizes. Future investigations should emphasize reporting on treatment safety.

Trial registration: PROSPERO CRD42014009506.

Keywords: Schizophrenia, Pediatric, Prolactin, Systematic review

\section{Background}

First-generation antipsychotics (FGAs), developed in the 1950s, were the first class of antipsychotics to be prescribed for schizophrenia. However, FGAs were associated with adverse events such as extrapyramidal motor control disabilities [1]. Second-generation antipsychotics (SGAs) were developed with the aim of providing similar or greater efficacy in the reduction of schizophreniarelated symptoms with a reduction in adverse events.

Of the men and women diagnosed with schizophrenia, $40 \%$ and $23 \%$, respectively, will be diagnosed before the age of 19 [2]. While SGAs have not been approved for use in the Canadian pediatric population [3], they are nevertheless widely prescribed for these patients $[4,5]$. Between 1999 and 2008 antipsychotic use in Canada for patients aged 18 years or younger increased from 1.9 per 1000 to 7.4 per 1000 [6]. However, while SGAs are considered a safer alternative to FGAs due to the reduced tendency to induce adverse neurological effects, other equally problematic adverse events have been associated with SGAs, especially in the pediatric population [7].

Hyperprolactinemia may become a clinical concern when patients are prescribed medications that block the inhibition of prolactin secretion, such as through interference with tuberoinfundibular dopamine pathway [8]. In 2011, the Canadian Alliance for Monitoring Effectiveness and Safety of Antipsychotics in Children published a report on the association between elevated prolactin levels and adverse events such as gynecomastia, galactorrhea, menstrual irregularities, sexual dysfunction, and decreased libido [9]. The effect of prolactin on gynecomastia in men and galactorrhea in women has also been reported elsewhere [10].

The effect of antipsychotics to raise prolactin levels have been summarized in a review by Byerly et al. [11]. Risperidone, in particular, has been associated with a greater than normal elevation in prolactin levels compared to other SGAs in both pediatric and adult populations [11-15]. Despite lack of approval, there is considerable off-label use of risperidone in Canadian pediatric patients [16].

The primary objective of this review is to examine the risk of prolactin-related adverse events associated with the use of antipsychotic medications for the treatment of schizophrenia and schizophrenia spectrum disorders in pediatric patients. The secondary objective is to examine changes in prolactin levels associated with the use of antipsychotic medications in this patient population.

\section{Methods}

\section{Search strategy}

The systematic review protocol (PROSPERO CRD42014009506) has been described elsewhere [17]. Briefly, clinical literature databases were systematically searched to identify randomized controlled trials (RCTs) and observational studies evaluating changes in prolactin levels and prolactin-related adverse events in pediatric patients, aged 5 to 18 years, diagnosed with schizophrenia or schizophrenia spectrum disorders and treated with first- or second-generation antipsychotics. For studies that included a control group, eligible comparators included first- and second-generation antipsychotics or placebo. The databases searched include: Medline, Embase, Cochrane Central Register for Controlled Trials, PsycINFO, ClinicalTrials.gov, the International Clinical Trials Registry Platform, and the Drug Industry Document Archive.

\section{Study selection}

Two reviewers, working independently, scanned all abstracts and relevant material identified in the literature search. The same two reviewers independently reviewed relevant abstracts in full-text. Discrepancies between the studies selected by the two reviewers were resolved by consensus. When necessary, a third reviewer was consulted.

\section{Data extraction and quality assessment}

Two reviewers working independently extracted data on study characteristics, interventions, patient characteristics at baseline, and outcomes for the study populations of interest for the final list of selected eligible studies. Study characteristics extracted included study design, inclusion and exclusion criteria, active treatment duration, and study quality items. Intervention characteristics extracted included dose, frequency of administration, duration, and concomitant/background therapies. 
Patient baseline characteristics extracted included age, sex, ethnicity, baseline prolactin levels (for males, females, and both sexes combined), previous antipsychotic use, age of schizophrenia onset, years since diagnosis, age at diagnosis, and schizophrenia subtype. Baseline disease severity scores from the Positive and Negative Syndrome Scale (PANSS) and the Brief Psychiatric Rating Scale (BPRS) were also extracted. Discrepancies between reviewers were resolved by involving a third reviewer and coming to a consensus.

For included RCTs, the quality of individual trials was assessed using the Risk of Bias instrument endorsed by the Cochrane Collaboration [18]. This instrument is used to evaluate 7 key domains: sequence generation, allocation concealment, blinding of participants and personnel, blinding of outcome assessors, incomplete outcome data, selective outcome reporting, and other sources of bias. For included observational studies, the quality of individual studies was assessed using the Newcastle-Ottawa Scale (NOS) [19]. This instrument is used to assess selection of patients, comparability of cohorts, and adequacy of outcomes and exposures.

\section{Data synthesis and analysis}

Data across studies were compared to identify possible trends between treatments with regards to incidence of adverse events and changes in prolactin levels from baseline in a narrative review. RCT and observational evidence were examined separately. Data for both study designs were extracted as either binary (prolactin-related adverse events) or continuous (change in prolactin levels). Data on change in prolactin levels were extracted for both males and females separately as well as one aggregate group (males and females together). In the absence of estimates for mean change form baseline in prolactin levels, this was calculated where baseline prolactin levels and endpoint prolactin levels were presented by subtraction of the means (calculation of the confidence interval by pooling of the standard errors of the two estimates). Where mean and confidence intervals of change in prolactin levels were provided for two of the three groups, both mean and standard error (which was used to calculate the confidence interval) were back-calculated to obtain estimates for the third group (for instance, if mean change in prolactin levels for both males and females aggregated was presented as well as data on males, this could be used to backcalculate estimates for females). Pooled proportions were calculated where multiple studies assessed the same treatment regimen and were calculated as the backtransformation of the weighted mean of the transformed proportions, as outlined by Miller 1978 [20]. Finally, forest plots were created depicting extracted data from RCTs (not created for data from observational studies due to expected heterogeneity in treatment duration between studies). These depict differences in mean change from baseline in prolactin levels between treatments. Forest plots and pooled proportions summarizing relevant outcome data were created in StatsDirect (Version 2.8.0).

\section{Results}

Of the 15,184 abstracts identified, 1,075 were evaluated at the full-text level. During the abstract screening phase, the most common reasons for exclusion were: studies were systematic reviews (classified as excluded for study design); studies were duplicates (classified as excluded for other); and studies assessed a population over 18 years of age (classified as excluded for population). The most common reason for exclusion in the full-text screening phase was a population over 18 years of age (classified as excluded for population). Eleven eligible studies were included, consisting of six RCTs and five non-randomized observational studies. Study flow is presented in Fig. 1.

\section{Evidence from RCTs}

All six included RCTs evaluated the use of SGAs [21-26]. The interventions assessed by these studies include four dose ranges of risperidone (1-3 mg/day, $4-6 \mathrm{mg} /$ day, $1.5-6 \mathrm{mg} /$ day, and $0.15-0.6 \mathrm{mg} /$ day) $[23,26]$, two doses of quetiapine $(400 \mathrm{mg} /$ day and $800 \mathrm{mg} /$ day $)$ [21], two doses of aripiprazole (10 $\mathrm{mg} /$ day and $30 \mathrm{mg} /$ day) [22], olanzapine $2.5-20 \mathrm{mg} /$ day [24], and three doses of paliperidone $(1.5 \mathrm{mg} /$ day, $3-6 \mathrm{mg} /$ day, and $6-12 \mathrm{mg} /$ day $)$ [25]. All RCTs, with the exception of Haas et al. 2009b, included a placebo control arm [26]. Characteristics of included RCTs are presented in Table 1.

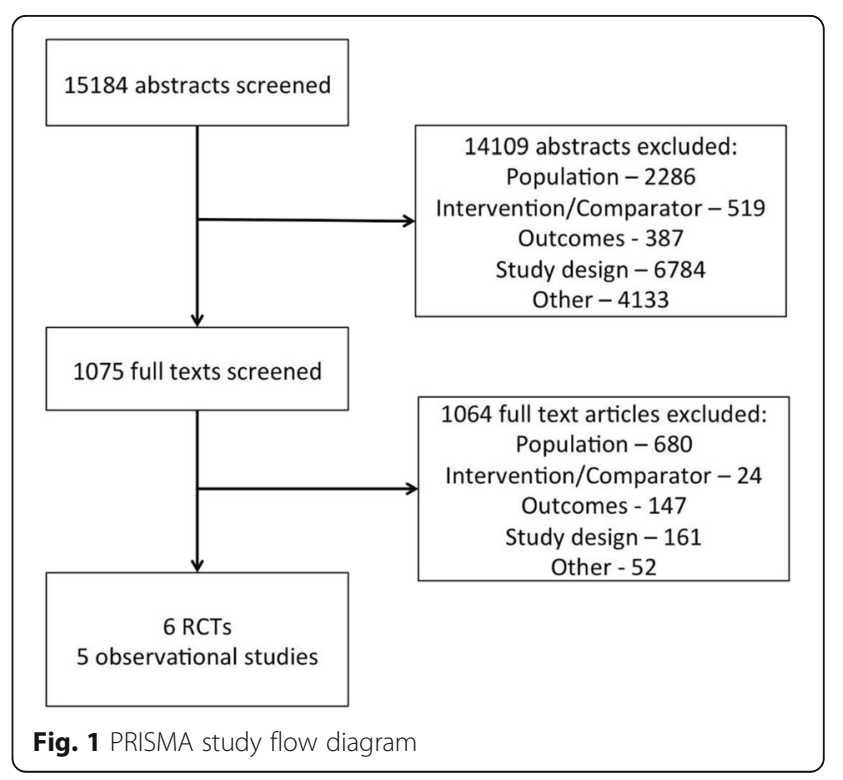


Table 1 Characteristics of included studies

\begin{tabular}{|c|c|c|c|c|}
\hline Study & Diagnosis (criteria for diagnosis) & Treatment(s) & Treatment duration, weeks & Inclusion criteria \\
\hline \multicolumn{5}{|c|}{ Randomized controlled trials } \\
\hline $\begin{array}{l}\text { Findling et al. } \\
2012 \text { [21] }\end{array}$ & $\begin{array}{l}\text { Schizophrenia } \\
\text { (DSM-IV-TR + K-SADS-PL) }\end{array}$ & $\begin{array}{l}\text { Quetiapine } 400 \text { mg/day } \\
\text { Quetiapine } 800 \text { mg/day } \\
\text { Placebo }\end{array}$ & 6 & $\begin{array}{l}\text { Inpatients or outpatients } \\
\text { aged } 13-17 \text { years; } \\
\text { PANSS } \geq 60\end{array}$ \\
\hline $\begin{array}{l}\text { Findling et al. } \\
2008 \text { [22] }\end{array}$ & $\begin{array}{l}\text { Schizophrenia } \\
\text { (DSM-IV) }\end{array}$ & $\begin{array}{l}\text { Aripiprazole } 10 \mathrm{mg} / \text { day } \\
\text { Aripiprazole } 30 \mathrm{mg} / \text { day } \\
\text { Placebo }\end{array}$ & 6 & $\begin{array}{l}\text { Aged } 13-17 \text { years; } \\
\text { PANSS } \geq 70\end{array}$ \\
\hline $\begin{array}{l}\text { Haas et al. } \\
\text { 2009a [23] }\end{array}$ & $\begin{array}{l}\text { Schizophrenia } \\
\text { (DSM-IV + K-SADS-PL) }\end{array}$ & $\begin{array}{l}\text { Risperidone 1-3 mg/day } \\
\text { Risperidone 4-6 mg/day } \\
\text { Placebo }\end{array}$ & 6 & $\begin{array}{l}\text { Aged } 13-17 \text { years; } \\
\text { PANSS } 60-120\end{array}$ \\
\hline $\begin{array}{l}\text { Haas et al. } \\
\text { 2009b [26] }\end{array}$ & $\begin{array}{l}\text { Schizophrenia } \\
\text { (DSM-IV + K-SADS-PL) }\end{array}$ & $\begin{array}{l}\text { Risperidone } 1.5-6 \mathrm{mg} / \text { day } \\
\text { Risperidone } 0.15-0.6 \mathrm{mg} / \text { day }\end{array}$ & 8 & $\begin{array}{l}\text { Aged } 13-17 \text { years; } \\
\text { PANSS } 60-120\end{array}$ \\
\hline $\begin{array}{l}\text { Kryzhanovskaya et al. } \\
2009 \text { [24] }\end{array}$ & $\begin{array}{l}\text { Schizophrenia } \\
\text { (DSM-IV-TR) }\end{array}$ & $\begin{array}{l}\text { Olanzapine } 2.5-20 \text { mg/day } \\
\text { Placebo }\end{array}$ & 6 & $\begin{array}{l}\text { Aged } 13-17 \text { years; } \\
\text { BPRS } \geq 35\end{array}$ \\
\hline $\begin{array}{l}\text { Singh et al. } \\
2011[25]\end{array}$ & $\begin{array}{l}\text { Schizophrenia } \\
\text { (DSM-IV + K-SADS-PL) }\end{array}$ & $\begin{array}{l}\text { Paliperidone } 1.5 \mathrm{mg} / \text { day } \\
\text { Paliperidone } 3-6 \mathrm{mg} / \text { day } \\
\text { Paliperidone } 6-12 \mathrm{mg} / \text { day } \\
\text { Placebo }\end{array}$ & 6 & $\begin{array}{l}\text { Aged } 12-17 \text { years; } \\
\text { Weight } \geq 29 \mathrm{~kg} ; \\
\text { PANSS } 60-120\end{array}$ \\
\hline \multicolumn{5}{|l|}{ Observational studies } \\
\hline $\begin{array}{l}\text { Duval et al. } \\
2008[27]\end{array}$ & $\begin{array}{l}\text { Schizophreniform disorder } \\
\text { (DSM-IV) }\end{array}$ & Risperidone 1-6 mg/day & 3 & Aged $14-18$ years \\
\hline $\begin{array}{l}\text { Kumra et al. } \\
2008[28]\end{array}$ & $\begin{array}{l}\text { Schizophrenia, schizoaffective } \\
\text { disorder (K-SADS-PL) }\end{array}$ & $\begin{array}{l}\text { Clozapine } 25-900 \text { mg/day } \\
\text { Olanzapine } 30 \text { mg/day }\end{array}$ & 12 & Aged $10-18$ years \\
\hline $\begin{array}{l}\text { Pandina et al. } \\
2012 \text { [29] }\end{array}$ & $\begin{array}{l}\text { Schizophrenia } \\
\text { (DSM-IV + K-SADS-PL) }\end{array}$ & Risperidone $2-6 \mathrm{mg} /$ day $^{\mathrm{a}}$ & 24 or $48^{\mathrm{a}}$ & $\begin{array}{l}\text { Aged } 13-17 \text { years; } \\
\text { PANSS } 40-120\end{array}$ \\
\hline $\begin{array}{l}\text { Ruan et al. } \\
2010 \text { [30] }\end{array}$ & $\begin{array}{l}\text { Schizophrenia } \\
\text { (DSM-IV-TR) }\end{array}$ & Risperidone $25-50$ mg biweekly & 24 & $\begin{array}{l}\text { Aged } 13-18 \text { years; } \\
\text { PANSS } \leq 80\end{array}$ \\
\hline $\begin{array}{l}\text { Schimmelmann et al. } \\
2007 \text { [31] }\end{array}$ & $\begin{array}{l}\text { Schizophrenia, schizoaffective } \\
\text { and schizophreniform disorder } \\
\text { (DSM-IV) }\end{array}$ & Quetiapine $200-800$ mg/day & 12 & $\begin{array}{l}\text { Aged } 12-17 \text { years; } \\
\text { PANSS } \geq 60\end{array}$ \\
\hline
\end{tabular}

BPRS Brief psychiatric rating scale, DSM Diagnostic and Statistical Manual of Mental Disorders, K-SADS-PL Kiddie Schedule for Affective Disorders and Schizophrenia for School-Age Children - Present and Lifetime Version, PANSS Positive and negative syndrome scale, TR text revision

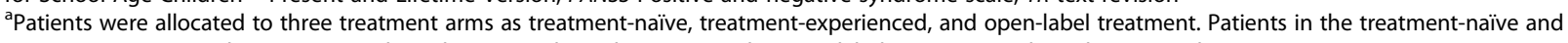
treatment-experienced arms were evaluated at 24 weeks and patients in the open-label arm were evaluated at 48 weeks

Treatment duration for most studies was 6 weeks, with the exception of Haas et al. 2009b which reported outcomes after an 8 week treatment duration [26]. All RCTs included adolescent patients, with age at enrollment restrictions varying between 12 and 18 years. Three RCTs only included patients with a PANSS score between 60 and $120[23,25,26]$ while one study included any patients with a PANSS score of 60 or above [21] and one study included patients with a PANSS score of 70 or above [22].

\section{Baseline patient characteristics}

Patient baseline characteristics are presented in Table 2. Baseline characteristics were generally well balanced, both between treatment arms and trials. Mean age varied between 15.1 and 16.3 years for all included RCTs and the percentage of males varied between $45 \%$ and $73 \%$. The distribution of males between study arms differed significantly for two trials, one reporting on the effects of aripiprazole, with percentages between $45 \%$ and $64 \%$ for the three arms [22], and the other reporting on paliperidone, with percentages between $45 \%$ and $70 \%$ for the four trial arms [25]. The majority of the study populations consisted of Caucasians, with all other races and ethnicities making up less than one quarter of each study arm in most studies. In the study by Haas et al. 2009a, however, Asian patients comprised over a third of the study population.

Of the three RCTs reporting on previous antipsychotic use, two reported percentages of patients with previous antipsychotic use above $70 \%$ in all arms $[24,25]$ and one reported previous antipsychotic use of $46 \%, 53 \%$, and $46 \%$ in the placebo arm, aripiprazole $10 \mathrm{mg} /$ day arm, and aripiprazole $30 \mathrm{mg} /$ day arm, respectively [22].

Three RCTs reported mean age of onset of symptoms related to schizophrenia, which varied between 12.5 and 14.2 years of age $[22,24,26]$. Three RCTS reported mean age at diagnosis of schizophrenia, which was also 
Table 2 Summary of baseline characteristics

\begin{tabular}{|c|c|c|c|c|c|c|c|c|c|c|c|}
\hline Study & $\mathrm{N}(\mathrm{ITT})$ & Treatment & $\begin{array}{l}\text { Age, mean } \\
(\mathrm{SD})\end{array}$ & Male, n (\%) & $\begin{array}{l}\text { Caucasian, } \\
\mathrm{n}(\%)\end{array}$ & $\begin{array}{l}\text { Baseline prolactin, } \\
\text { both sexes, }(\mathrm{ng} / \mathrm{mL}) \text {, } \\
\text { mean (SD) }\end{array}$ & $\begin{array}{l}\text { Baseline prolactin, } \\
\text { males, }(\mathrm{ng} / \mathrm{mL}) \text {, } \\
\text { mean }(\mathrm{SD})\end{array}$ & $\begin{array}{l}\text { Baseline prolactin, } \\
\text { females, }(\mathrm{ng} / \mathrm{mL} \text { ), } \\
\text { mean (SD) }\end{array}$ & $\begin{array}{l}\text { Previous } \\
\text { antipsychotic } \\
\text { use, } \mathrm{n}(\%)\end{array}$ & $\begin{array}{l}\text { Proportion diagnosed } \\
\text { as paranoid } \\
\text { schizophrenia, n (\%) }\end{array}$ & $\begin{array}{l}\text { Baseline } \\
\text { PANSS score, } \\
\text { mean (SD) }\end{array}$ \\
\hline \multicolumn{12}{|c|}{ Randomized controlled trials } \\
\hline \multirow{3}{*}{$\begin{array}{l}\text { Findling et al. } \\
2012 \text { [21] }\end{array}$} & 73 & Quetiapine 400 mg/day & $15.5(1.3)$ & $43(58.9)$ & $45(61.6)$ & $20.8(17.0)$ & NR & NR & NR & NR & $96.2(17.7)$ \\
\hline & 74 & Quetiapine 800 mg/day & $15.5(1.3)$ & $44(59.5)$ & $44(59.5)$ & $18.1(20.1)$ & NR & NR & $N R$ & NR & $97.0(15.3)$ \\
\hline & 73 & Placebo & $15.3(1.4)$ & $42(57.5)$ & $46(63.0)$ & $28.7(29.1)$ & NR & NR & $N R$ & NR & $96.7(18.0)$ \\
\hline \multirow{3}{*}{$\begin{array}{l}\text { Findling et al. } \\
2008 \text { [22] }\end{array}$} & 100 & Aripiprazole 10 mg/day & $15.6(1.3)$ & $45(45.0)$ & $5] 4(54.0)$ & NR & NR & $N R$ & $53(53.0)$ & NR & $93.7(15.7)$ \\
\hline & 102 & Aripiprazole 30 mg/day & $15.4(1.4)$ & $65(63.7)$ & $62(60.8)$ & NR & NR & NR & $47(46.1)$ & NR & $94.9(15.5)$ \\
\hline & 100 & Placebo & $15.4(1.4)$ & $61(61.0)$ & $64(64.0)$ & NR & NR & NR & $46(46.0)$ & NR & $95.0(15.5)$ \\
\hline \multirow{3}{*}{$\begin{array}{l}\text { Haas et al. } \\
\text { 2009a [23] }\end{array}$} & 55 & Risperidone 1-3 mg/day & $15.7(1.3)$ & $30(54.5)$ & $33(60.0)$ & $29.6(38.2)$ & $21.6(15.2)$ & $39.2(53.0)$ & NR & $38(69.1)$ & NR \\
\hline & 51 & Risperidone 4-6 mg/day & $15.7(1.3)$ & $37(72.5)$ & $24(47.1)$ & $24.1(23.0)$ & $22.7(19.9)$ & $27.9(28.3)$ & NR & $34(66.7)$ & NR \\
\hline & 54 & Placebo & $15.5(1.4)$ & $35(64.8)$ & $27(50.0)$ & $22.6(23.3)$ & $21.5(21.1)$ & $24.6(25.9)$ & NR & $38(70.4)$ & NR \\
\hline \multirow{2}{*}{$\begin{array}{l}\text { Haas et al. } \\
2009 \mathrm{~b}[26]\end{array}$} & 125 & Risperidone $1.5-6 \mathrm{mg} /$ day & $15.6(1.3)$ & $65(52.0)$ & $104(83.2)$ & NR & NR & NR & NR & $83(66.4)$ & $96.4(15.4)$ \\
\hline & 132 & Risperidone $0.15-0.6 \mathrm{mg} / \mathrm{day}$ & $15.6(1.3)$ & $80(60.6)$ & $111(84.9)$ & NR & NR & NR & NR & $92(69.7)$ & $93.3(14.1)$ \\
\hline \multirow{2}{*}{$\begin{array}{l}\text { Kryzhanovskaya et al. } \\
2009 \text { [24] }\end{array}$} & 72 & Olanzapine $2.5-20$ mg/day & $16.1(1.3)$ & $51(70.8)$ & $52(72.2)$ & $15.6(12.3)$ & NR & NR & $51(70.8)$ & NR & $95.3(14.1)$ \\
\hline & 35 & Placebo & $16.3(1.6)$ & $24(68.6)$ & $25(71.4)$ & $18.7(16.2)$ & NR & NR & $30(85.7)$ & NR & $95.5(14.1)$ \\
\hline \multirow{4}{*}{$\begin{array}{l}\text { Singh et al. } \\
2011 \text { [25] }\end{array}$} & 54 & Paliperidone $1.5 \mathrm{mg} /$ day & $15.1(1.5)$ & $30(55.6)$ & $35(64.8)$ & $25.1(32.8)$ & $14.7(14.5)$ & $38.0(45.5)$ & $47(87.0)$ & NR & $91.6(12.5)$ \\
\hline & 48 & Paliperidone 3-6 mg/day & $15.3(1.6)$ & $31(64.6)$ & $34(70.8)$ & $26.6(43.2)$ & $16.1(1.8)$ & $45.67(71.0)$ & $44(91.7)$ & NR & $90.6(14.0)$ \\
\hline & 47 & Paliperidone 6-12 mg/day & $15.5(1.6)$ & $33(70.2)$ & $32(68.1)$ & $20.8(22.3)$ & $18.2(13.7)$ & $26.88(33.9)$ & $40(85.1)$ & NR & $91.5(13.9)$ \\
\hline & 51 & Placebo & $15.7(1.4)$ & $23(45.1)$ & $35(68.6)$ & $24.5(30.5)$ & $13.6(13.6)$ & $33.43(38.5)$ & $48(94.1)$ & NR & $90.6(12.1)$ \\
\hline \multicolumn{12}{|l|}{ Observational studies } \\
\hline $\begin{array}{l}\text { Duval et al. } \\
2008 \text { [27] }\end{array}$ & 16 & Risperidone 1-6 mg/day & $15.7(1.3)$ & $10(62.5)$ & $16(100.0)$ & $16(9)$ & NR & NR & NR & NR & NR \\
\hline \multirow{2}{*}{$\begin{array}{l}\text { Kumra et al. } \\
2008 \text { [28] }\end{array}$} & 14 & Clozapine 25-900 mg/day & $15.3(2.3)$ & $5(35.7)$ & $1(7.1)$ & NR & NR & NR & $14(100.0)$ & NR & NR \\
\hline & 19 & Olanzapine 30 mg/day & $15.6(1.7)$ & $11(57.9)$ & $5(26.3)$ & NR & NR & NR & $19(100.0)$ & NR & NR \\
\hline \multirow[t]{3}{*}{$\begin{array}{l}\text { Pandina et al. } \\
2012 \text { [29] }\end{array}$} & 48 & $\begin{array}{l}\text { Risperidone } 2-6 \text { mg/day } \\
\text { (treatment-naiive) }\end{array}$ & $15.4(1.4)$ & $30(62.5)$ & $25(52.1)$ & $17.0(20.4)$ & $18.6(24.7)$ & $14.3(6.7)$ & NR & $33(68.8)$ & $84.7(16.8)$ \\
\hline & 292 & $\begin{array}{l}\text { Risperidone } 2-6 \text { mg/day } \\
\text { (treatment-experienced) }\end{array}$ & $15.5(1.7)$ & $178(61.0)$ & $218(74.7)$ & $53.4(33.9)$ & $40.4(26.3)$ & $73.6(43.0)$ & $292(100.0)$ & $195(66.8)$ & $72.1(19.4)$ \\
\hline & 50 & $\begin{array}{l}\text { Risperidone } 2-6 \text { mg/day } \\
\text { (open-label) }\end{array}$ & $15.5(1.4)$ & $30(60.0)$ & $42(84.0)$ & $73.6(44.9)$ & $55.8(25.3)$ & $100.3(62.3)$ & NR & $35(70.0)$ & $83.9(13.5)$ \\
\hline $\begin{array}{l}\text { Ruan et al. } \\
2010 \text { [30] }\end{array}$ & 31 & $\begin{array}{l}\text { Risperidone } 25-50 \mathrm{mg} \\
\text { biweekly }\end{array}$ & $15.9(3.3)$ & $13(41.9)$ & NR & $54.8(17.7)$ & NR & NR & NR & $24(77.4)$ & $57.8(1.8)$ \\
\hline $\begin{array}{l}\text { Schimmelmann et al. } \\
2007 \text { [31] }\end{array}$ & 56 & Quetiapine $200-800$ mg/day & $15.9(1.3)$ & $38(67.9)$ & $47(83.9)$ & $15.9(23.3)$ & $12.6(6.3)$ & $22.9(41.4)$ & $13(23.2)$ & NR & $91.5(17.2)$ \\
\hline
\end{tabular}

ITT Intention to treat, $N R$ Not reported, SD Standard deviation 
similar across trials, varying from 12.5 years of age to 15.3 years of age $[23,25,26]$. In addition, two three-arm studies reported mean years since schizophrenia diagnosis, which was similar in all three arms for both studies [21, 22]. Three RCTs reported the proportion of patients diagnosed under each schizophrenia subtype $[21,23,26]$. In all of these studies, the majority of patients were diagnosed with paranoid schizophrenia, followed by undifferentiated schizophrenia and disorganized schizophrenia $[23,26]$. No study reported age at first psychosis.

Baseline disease severity assessments were consistent across studies. Most RCTs reported baseline disease severity using the PANSS, with mean values varying from 90.6 to 97.0 [21, 22, 24-26]. One RCT reported mean baseline BPRS scores of 50.3 and 50.1 for the olanzapine $2.5-20 \mathrm{mg} /$ day and placebo arms, respectively [24].

Two studies reported overall population baseline prolactin levels, with Findling et al. (2012) reporting mean baseline values between $18.1 \mathrm{ng} / \mathrm{mL}$ and $28.7 \mathrm{ng} / \mathrm{mL}$ and Kryzhanovskaya et al. (2009) reporting mean baseline values between $15.6 \mathrm{ng} / \mathrm{mL}$ and $18.7 \mathrm{ng} / \mathrm{mL}$ [21, 24]. Additionally, two trials reported baseline prolactin levels for both males and females separately. For males, Haas et al. (2009a) reported mean baseline values from $21.5 \mathrm{ng} / \mathrm{mL}$ and $22.7 \mathrm{ng} / \mathrm{mL}$, while Singh et al. (2011) reported values between $13.6 \mathrm{ng} / \mathrm{mL}$ and $18.2 \mathrm{ng} / \mathrm{mL}$ [23, 25]. Baseline prolactin levels were higher in females, but similar across studies, as Haas et al. (2009a) reported values between $24.6 \mathrm{ng} / \mathrm{mL}$ and $39.2 \mathrm{ng} / \mathrm{mL}$, while Singh et al. (2011) reported values between $26.9 \mathrm{ng} / \mathrm{mL}$ and $45.7 \mathrm{ng} / \mathrm{mL}$ [23, 25]. Overall population baseline prolactin levels were calculated for these two studies.

\section{Outcomes}

Prolactin-related adverse events Prolactin-related adverse events were sparsely reported (see Table 3 ). Three RCTs reported overall prolactin-related adverse events, gynecomastia/galactorrhea, and amenorrhea/dysmenorrhea in patients treated with varying doses of aripiprazole and risperidone [22, 23, 26]. In two studies, which assessed aripiprazole and risperidone, no cases of prolactin-related adverse events were reported [22, 23]. A study comparing doses of risperidone of $1.5-6 \mathrm{mg} /$ day and $0.15-0.6 \mathrm{mg} /$ day reported seven events $(5.6 \%$ of study patients) and two events (1.5\%), respectively [26]. Specifically, investigators reported three (2.4\%) and two $(1.5 \%)$ cases of gynecomastia/galactorrhea in the risperidone $1.5-6 \mathrm{mg} /$ day and risperidone $0.15-0.6 \mathrm{mg} /$ day arms, respectively, as well as a single case $(1.7 \%$ of female patients) of amenorrhea/dysmenorrhea in the risperidone $1.5-6 \mathrm{mg} /$ day $\mathrm{arm}$.
Change in prolactin levels: Change from baseline, treatment-placebo comparison Five RCTs provided data for mean change in prolactin levels for the overall population (see Table 4) [21-25]. Mean changes from baseline compared to placebo for each intervention in the overall population are presented in Fig. 2.

Compared to baseline, both doses of quetiapine and aripiprazole resulted in decreased prolactin levels. When compared to placebo, these changes were significantly greater compared to baseline for quetiapine but not for aripiprazole.

Both doses of risperidone, olanzapine, and paliperidone $3-5 \mathrm{mg} /$ day and $6-12 \mathrm{mg} /$ day were found to increase prolactin levels from baseline. These changes also reflected statistically significant increases when compared to placebo. Paliperidone $1.5 \mathrm{mg} /$ day was not associated with a statistically significant change.

Three RCTs reported changes in serum prolactin levels stratified by sex $[21,23,25]$. When evaluating the change from baseline for each of the included SGAs, trends reflected those observed in the overall populations for both males and females. However, the change from baseline for females treated with any dose range of risperidone was higher than males treated with the same dose. Figures 3 and 4 present the mean changes from baseline compared to placebo for males and females, respectively. Compared to placebo, the change from baseline for females treated with either dose of quetiapine resulted in a statistically significant difference. This change was not found to be significant in males. Compared to placebo, both males and females were found to experience significant increases when treated with risperidone, though this increase was more marked in female patients treated with risperidone 4-6 mg/day. Conversely, the change from baseline compared to placebo was significant for males, but not females, treated with paliperidone $3-6 \mathrm{mg} /$ day and 6-12 $\mathrm{mg} /$ day.

Change in prolactin levels: Change from baseline, treatment-treatment comparison Figure 2 presents comparisons for change from baseline in prolactin levels between treatments for the overall population. Risperidone $4-6 \mathrm{mg} /$ day reported a greater increase in prolactin levels when compared with the lower dose of 1-3 mg/day. Similarly, both paliperidone $3-6 \mathrm{mg} /$ day and $6-12 \mathrm{mg} /$ day reported a greater increase in prolactin levels when compared with paliperidone $1.5 \mathrm{mg} /$ day. However, the difference between paliperidone $6-12 \mathrm{mg} /$ day and $3-6 \mathrm{mg} /$ day was not statistically significant. Further, no statistically significant differences were reported for the comparisons between quetiapine $400 \mathrm{mg} /$ day and $800 \mathrm{mg} /$ day or between aripiprazole $30 \mathrm{mg} /$ day and $10 \mathrm{mg} /$ day. 
Table 3 Prolactin-related adverse events

\begin{tabular}{|c|c|c|c|c|c|c|}
\hline Study & $N(I T T)$ & Treatment & $\begin{array}{l}\text { Prolactin-related } \\
\text { adverse events, } \\
\mathrm{n}(\%)\end{array}$ & $\begin{array}{l}\text { Gynecomastia/ } \\
\text { galactorrhea, } \\
\mathrm{n}(\%)\end{array}$ & $\begin{array}{l}\text { Amenorrhea/ } \\
\text { dysmenorrhea, } \\
\mathrm{n}(\%)\end{array}$ & $\begin{array}{l}\text { Impotence/ } \\
\text { decreased libido, } \\
\mathrm{n}(\%)\end{array}$ \\
\hline \multicolumn{7}{|c|}{ Randomized controlled trials } \\
\hline \multirow{3}{*}{$\begin{array}{l}\text { Findling et al. } \\
2012 \text { [21] }\end{array}$} & 73 & Quetiapine 400 mg/day & $N R$ & NR & $N R$ & NR \\
\hline & 74 & Quetiapine 800 mg/day & NR & NR & $N R$ & NR \\
\hline & 73 & Placebo & $N R$ & NR & $N R$ & NR \\
\hline \multirow{3}{*}{$\begin{array}{l}\text { Findling et al. } \\
2008 \text { [22] }\end{array}$} & 100 & Aripiprazole 10 mg/day & $0(0.0)$ & NR & $N R$ & NR \\
\hline & 102 & Aripiprazole 30 mg/day & $0(0.0)$ & NR & $N R$ & NR \\
\hline & 100 & Placebo & $0(0.0)$ & NR & $N R$ & NR \\
\hline \multirow{3}{*}{$\begin{array}{l}\text { Haas et al. } \\
\text { 2009a [23] }\end{array}$} & 55 & Risperidone 1-3 mg/day & $0(0.0)$ & NR & $N R$ & NR \\
\hline & 51 & Risperidone 4-6 mg/day & $0(0.0)$ & NR & $N R$ & NR \\
\hline & 54 & Placebo & $0(0.0)$ & NR & NR & $N R$ \\
\hline \multirow{2}{*}{$\begin{array}{l}\text { Haas et al. } \\
2009 \mathrm{~b}[26]\end{array}$} & 125 & Risperidone 1.5-6 mg/day & $7(5.6)$ & $3(2.4)$ & $1(1.0)$ & NR \\
\hline & 132 & Risperidone $0.15-0.6$ mg/day & $2(1.5)$ & $2(1.5)$ & $0(0.0)$ & NR \\
\hline \multirow{2}{*}{$\begin{array}{l}\text { Kryzhanovskaya et al. } \\
2009 \text { [24] }\end{array}$} & 72 & Olanzapine $2.5-20$ mg/day & $N R$ & NR & $N R$ & NR \\
\hline & 35 & Placebo & $N R$ & $N R$ & $N R$ & NR \\
\hline \multirow{4}{*}{$\begin{array}{l}\text { Singh et al. } \\
2011[25]\end{array}$} & 54 & Paliperidone 1.5 mg/day & NR & NR & NR & NR \\
\hline & 48 & Paliperidone 3-6 mg/day & NR & NR & $N R$ & $N R$ \\
\hline & 47 & Paliperidone 6-12 mg/day & NR & NR & $N R$ & NR \\
\hline & 51 & Placebo & NR & NR & NR & NR \\
\hline \multicolumn{7}{|l|}{ Observational studies } \\
\hline $\begin{array}{l}\text { Duval et al. } \\
2008 \text { [27] }\end{array}$ & 16 & Risperidone 1-6 mg/day & NR & NR & NR & NR \\
\hline \multirow{2}{*}{$\begin{array}{l}\text { Kumra et al. } \\
2008 \text { [28] }\end{array}$} & 14 & Clozapine 25-900 mg/day & NR & NR & NR & NR \\
\hline & 19 & Olanzapine 30 mg/day & $N R$ & NR & $N R$ & NR \\
\hline \multirow{3}{*}{$\begin{array}{l}\text { Pandina et al. } \\
2012^{\mathrm{a}}[29]\end{array}$} & 48 & Risperidone 2-6 mg/day (treatment-naïve) & $36(9.3)$ & $14(3.6)$ & $3(1.0)$ & $1(<0.5)$ \\
\hline & 292 & $\begin{array}{l}\text { Risperidone } 2-6 \text { mg/day (treatment- } \\
\text { experienced) }\end{array}$ & & & & \\
\hline & 50 & Risperidone 2-6 mg/day (open-label) & & & & \\
\hline $\begin{array}{l}\text { Ruan et al. } \\
2010 \text { [30] }\end{array}$ & 31 & Risperidone 25-50 mg biweekly & $5(16.1)$ & $2(6.5)$ & $3(9.7)$ & NR \\
\hline $\begin{array}{l}\text { Schimmelmann et al. } \\
2007 \text { [31] }\end{array}$ & 56 & Quetiapine $200-800$ mg/day & NR & $3(5.4)$ & $4(7.1)$ & $9(16.1)$ \\
\hline
\end{tabular}

ITT Intent to treat, NR Not reported

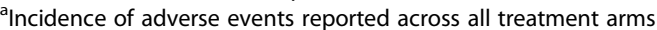

Figures 3 and 4 present comparisons of change in prolactin levels from baseline between treatments for the male and female populations, respectively. The comparison between risperidone at $4-6 \mathrm{mg} /$ day and $1-3 \mathrm{mg} /$ day was significant in the female population, but not in the male population, with the higher dose producing a greater change from baseline for both populations. Similarly, comparisons between quetiapine $800 \mathrm{mg} /$ day and $400 \mathrm{mg} /$ day, paliperidone $6-12 \mathrm{mg} /$ day and $1.5 \mathrm{mg} /$ day, and paliperidone $6-12 \mathrm{mg} /$ day and $1.5 \mathrm{mg} /$ day were statistically significant in the male population, but not in the female population, with greater increases observed in the treatments with the higher dose. The comparison between paliperidone 6-12 mg/day and paliperidone 3-6 mg/day was not significant for either patient population.

\section{Evidence from non-randomized, observational studies}

Five observational studies were identified which assessed patient experiences with SGAs [27-31]. The interventions represented in this set of literature included three doses of risperidone (1-6 mg/day, 2-6 mg/day, 25-50 mg biweekly) [27, 29, 30], quetiapine $200-800 \mathrm{mg} /$ day [31], clozapine $25-900 \mathrm{mg} /$ day [28], and olanzapine $30 \mathrm{mg} /$ day [28]. Characteristics of included observational studies are presented in Table 1. 
Table 4 Changes from baseline in prolactin

\begin{tabular}{|c|c|c|c|c|c|}
\hline Study & Treatment & $\begin{array}{l}\text { Time point, } \\
\text { weeks }\end{array}$ & $\begin{array}{l}\text { Prolactin change, both } \\
\text { sexes }(\mathrm{ng} / \mathrm{mL}) \text {, mean }(\mathrm{SD})\end{array}$ & $\begin{array}{l}\text { Prolactin change, males } \\
(\mathrm{ng} / \mathrm{mL}) \text {, mean (SD) }\end{array}$ & $\begin{array}{l}\text { Prolactin change, females } \\
(\mathrm{ng} / \mathrm{mL}) \text {, mean (SD) }\end{array}$ \\
\hline \multicolumn{6}{|c|}{ Randomized controlled trials } \\
\hline \multirow{3}{*}{$\begin{array}{l}\text { Findling et al. } \\
2012 \text { [21] }\end{array}$} & Quetiapine 400 mg/day & 6 & $-10.6(16.1)$ & $-9.22(14.4)$ & $-12.4(18.5)$ \\
\hline & Quetiapine 800 mg/day & 6 & $-7.8(26.5)$ & $-3.7(11.6)$ & $-14.0(39.1)$ \\
\hline & Placebo & 6 & $-18.3(28.8)$ & $-6.53(15.1)$ & $-33.9(34.9)$ \\
\hline \multirow{3}{*}{$\begin{array}{l}\text { Findling et al. } \\
2008 \text { [22] }\end{array}$} & Aripiprazole 10 mg/day & 6 & $-11.9(23.3)$ & NR & NR \\
\hline & Aripiprazole 30 mg/day & 6 & $-15.1(26.9)$ & NR & NR \\
\hline & Placebo & 6 & $-8.5(24.2)$ & NR & $N R$ \\
\hline \multirow{3}{*}{$\begin{array}{l}\text { Haas et al. } \\
\text { 2009a [23] }\end{array}$} & Risperidone 1-3 mg/day & 6 & $25.5(33.5)$ & $16(23.7)$ & $36.9(41.3)$ \\
\hline & Risperidone 4-6 mg/day & 6 & $49.5(46.9)$ & $26.4(28.5)$ & $77.3(60.8)$ \\
\hline & Placebo & 6 & $-5.9(24.9)$ & $-3.2(24.8)$ & $-9.2(24.1)$ \\
\hline \multirow{2}{*}{$\begin{array}{l}\text { Haas et al. } \\
\text { 2009b [26] }\end{array}$} & Risperidone $1.5-6$ mg/day & 8 & NR & NR & NR \\
\hline & Risperidone $0.15-0.6$ mg/day & 8 & NR & NR & NR \\
\hline \multirow{2}{*}{$\begin{array}{l}\text { Kryzhanovskaya et al. } \\
2009 \text { [24] }\end{array}$} & Olanzapine $2.5-20$ mg/day & 6 & $8.8(17.9)$ & NR & NR \\
\hline & Placebo & 6 & $-3.3(14.8)$ & NR & NR \\
\hline \multirow{4}{*}{$\begin{array}{l}\text { Singh et al. } \\
2011 \text { [25] }\end{array}$} & Paliperidone 1.5 mg/day & 6 & $3.3(36.0)$ & $3.6(19.1)$ & $2.9(48.6)$ \\
\hline & Paliperidone 3-6 mg/day & 6 & $22.7(34.1)$ & $22.8(30.1)$ & $22.4(38.7)$ \\
\hline & Paliperidone 6-12 mg/day & 6 & $22.4(35.5)$ & $21.3(31.1)$ & $24.9(42.0)$ \\
\hline & Placebo & 6 & $2.7(15.2)$ & $0.6(9.4)$ & $4.4(18.3)$ \\
\hline \multicolumn{6}{|l|}{ Observational studies } \\
\hline $\begin{array}{l}\text { Duval et al. } \\
2008[27]\end{array}$ & Risperidone 1-6 mg/day & 3 & $47.9(23.6)$ & NR & $N R$ \\
\hline \multirow{4}{*}{$\begin{array}{l}\text { Kumra et al. } \\
2008 \text { [28] }\end{array}$} & Clozapine 25-900 mg/day & 12 & NR & NR & $N R$ \\
\hline & Olanzapine 30 mg/day & 12 & NR & NR & NR \\
\hline & Clozapine 25-900 mg/day & 24 & NR & NR & NR \\
\hline & Olanzapine 30 mg/day & 24 & NR & NR & NR \\
\hline \multirow[t]{3}{*}{$\begin{array}{l}\text { Pandina et al. } \\
2012 \text { [29] }\end{array}$} & $\begin{array}{l}\text { Risperidone } 2-6 \text { mg/day } \\
\text { (treatment-naïve) }\end{array}$ & 24 & $66.8(41.8)$ & $29.1(32.6)$ & $83.4(44.7)$ \\
\hline & $\begin{array}{l}\text { Risperidone } 2-6 \text { mg/day } \\
\text { (treatment-experienced) }\end{array}$ & 24 & $-11.6(43.3)$ & $-6.2(22.2)$ & $-14.0(49.3)$ \\
\hline & $\begin{array}{l}\text { Risperidone 2-6 mg/day } \\
\text { (open-label) }\end{array}$ & 48 & $6.8(35.2)$ & $3.7(28.5)$ & $11.5(43.3)$ \\
\hline $\begin{array}{l}\text { Ruan et al. } \\
2010 \text { [30] }\end{array}$ & Risperidone 25-50 mg biweekly & 24 & $-13.1(17.1)$ & NR & NR \\
\hline $\begin{array}{l}\text { Schimmelmann et al. } \\
2007 \text { [31] }\end{array}$ & Quetiapine $200-800$ mg/day & 12 & $-1.4(21.1)$ & $-4.5(5.7)$ & $3.2(21.7)$ \\
\hline
\end{tabular}

NR Not reported, SD Standard deviation

Two of the observational studies restricted enrollment to patients with a diagnosis of only schizophrenia $[29,30]$. The remaining studies represented patients with schizophrenia, schizoaffective disorder, and schizophreniform disorder [27, 28, 31]. Three of the observational studies also specified disease severity in the inclusion criteria, with PANSS scores restricted to between 40 and 120 [29], 80 or below [30], and 60 or above [31].

Treatment durations varied widely, with one observational study reporting on patient outcomes after 3 weeks of treatment [27], two studies after 12 weeks [28, 31], one study after 24 weeks [30], and one three-arm study after 24 weeks for two arms and 48 weeks for the third arm [29]. All observational studies included adolescent patients with ages varying between 10 and 18 years.

One study, published by Pandina and colleagues (2012), adopted a non-conventional comparative observational study methodology [29]. This three-arm study was designed to include one arm with 24 weeks prior treatment experience with risperidone $2-6 \mathrm{mg} /$ day (treatment-experienced arm), one arm with no risperidone experience (treatment-naïve arm), and one arm 
Direct comparison

Risperidone 1-3 mg/day vs. Placebo Risperidone $4-6 \mathrm{mg} / \mathrm{day}$ vs. Placebo Quetiapine $400 \mathrm{mg} /$ day vs. Placebo Quetiapine 800 mglday vs. Placebo Aripiprazole $10 \mathrm{mg} /$ day vs. Placebo Aripiprazole $30 \mathrm{mg} /$ day vs. Placebo Olanzapine $2.5-20 \mathrm{mg} /$ day vs. Placebo Paliperidone $1.5 \mathrm{mg} /$ day vs. Placebo Paliperidone $3-6$ mg/day vs. Placebo Paliperidone 6-12 mg/day vs. Placebo Risperidone 4-6 mg/day vs. Risperidone $1-3 \mathrm{mg} / \mathrm{day}$ Quetiapine $800 \mathrm{mg} /$ day vs. Quetiapine $400 \mathrm{mg} / \mathrm{day}$ Aripiprazole $30 \mathrm{mg} /$ day vs. Aripiprazole $10 \mathrm{mg} / \mathrm{day}$ Paliperidone $3-6 \mathrm{mg} / \mathrm{day}$ vs. Paliperidone $1.5 \mathrm{mg} / \mathrm{day}$ Paliperidone 6-12 mg/day vs. Paliperidone $1.5 \mathrm{mg} /$ day Paliperidone 6-12 mg/day vs. Paliperidone 3-6 mg/day
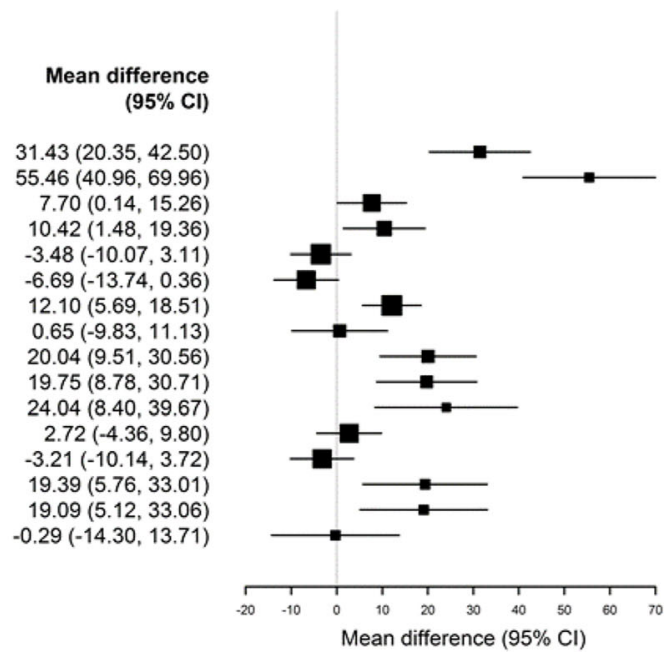

Fig. 2 Forest plot of mean change from baseline for SGAs presented in RCTs, overall population

with varying levels of treatment experience prior to study treatment (open-label arm). At enrollment, patients received 24 weeks open-label treatment with risperidone $2-6 \mathrm{mg} /$ day, thus resulting in one arm with 48 weeks of treatment experience and two arms with 24 weeks of treatment experience.

\section{Baseline patient characteristics}

Patient baseline characteristics are presented in Table 2. Mean ages were homogenous across studies, varying between 15.3 and 15.9 years. However, the distribution of males and females varied between trials, with percentages of males in each study arm ranging from $36 \%$ to $68 \%$. Race was reported in four observational studies $[27-29,31]$. In two cases, patient populations were comprised either primarily or entirely of Caucasians [27, 31]. Imbalances were reported in the three-arm study, with the percentage of Caucasian patients ranging from $52 \%$ to $84 \%$ across arms [29]. The study by Kumra et al.
(2008) comprised a mixed race population with AfricanAmericans representing the largest percentage of patients at $36 \%$ and $47 \%$ across treatment arms [28].

Where reported, previous antipsychotic treatment experience varied from $100 \%$ of patients $[28,29]$ to less than $25 \%$ [31]. Similarly, disease severity was not consistent across the included studies. As assessed by the PANSS, mean reported scores varied from 57.8 to 91.5 . A single study reported mean age of schizophrenia symptoms of 12.7 and 11.7 for the two treatment arms [32]. Mean age of diagnosis of schizophrenia or schizophrenia spectrum disorder was reported in a single study, with ages varying between 14.8 and 15.1 across study arms [29]. Two observational studies reported schizophrenia subtype and in both cases the majority of patients were diagnosed with paranoid schizophrenia $[29,30]$. Overall mean baseline prolactin levels varied between studies from a minimum of $15.9 \mathrm{ng} / \mathrm{mL}$ to a maximum of $73.6 \mathrm{ng} / \mathrm{mL}$. In two observational studies,
Direct comparison

Risperidone $1-3 \mathrm{mg} / \mathrm{day}$ vs. Placebo Risperidone $4-6 \mathrm{mg} /$ day vs. Placebo Quetiapine $400 \mathrm{mg} /$ day vs. Placebo Quetiapine $800 \mathrm{mg} /$ day vs. Placebo Paliperidone $1.5 \mathrm{mg} /$ day vs. Placebo Paliperidone $3-6 \mathrm{mg} /$ day vs. Placebo Paliperidone 6-12 mg/day vs. Placebo Risperidone $4-6 \mathrm{mg} / \mathrm{day}$ vs. Risperidone $1-3 \mathrm{mg} / \mathrm{day}$ Quetiapine $800 \mathrm{mg} /$ day vs. Quetiapine $400 \mathrm{mg} / \mathrm{day}$ Quetiapine $800 \mathrm{mg} /$ day vs. Quetiapine $400 \mathrm{mg} /$ day Paliperidone $3-6 \mathrm{mg} /$ day vs. Paliperidone $1.5 \mathrm{mg} /$ day
Paliperidone $6-12 \mathrm{mg} /$ day vs. Paliperidone $1.5 \mathrm{mg} /$ day Paliperidone $6-12 \mathrm{mg} /$ day vs. Paliperidone $3-6 \mathrm{mg} /$ day $-1.50(-16.50,13.50)$ $29.60(17.28,41.92)$ $2.83(-2.89,8.55)$ $3.05(-4.79,10.89)$ $20.77(9.49,32.05)$ $0.40(-210,22.90)$ $19.22(6.61,31.83)$
Mean difference

$(95 \% \mathrm{Cl})$

$19.20(7.39,31.01)$ $-2.69(-8.97,3.59)$ $22.27(11.00,33.54)$ $5.52(0.02,11.02)$

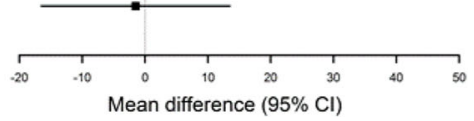

Fig. 3 Forest plot of mean change from baseline for SGAs presented in RCTs, male population only 

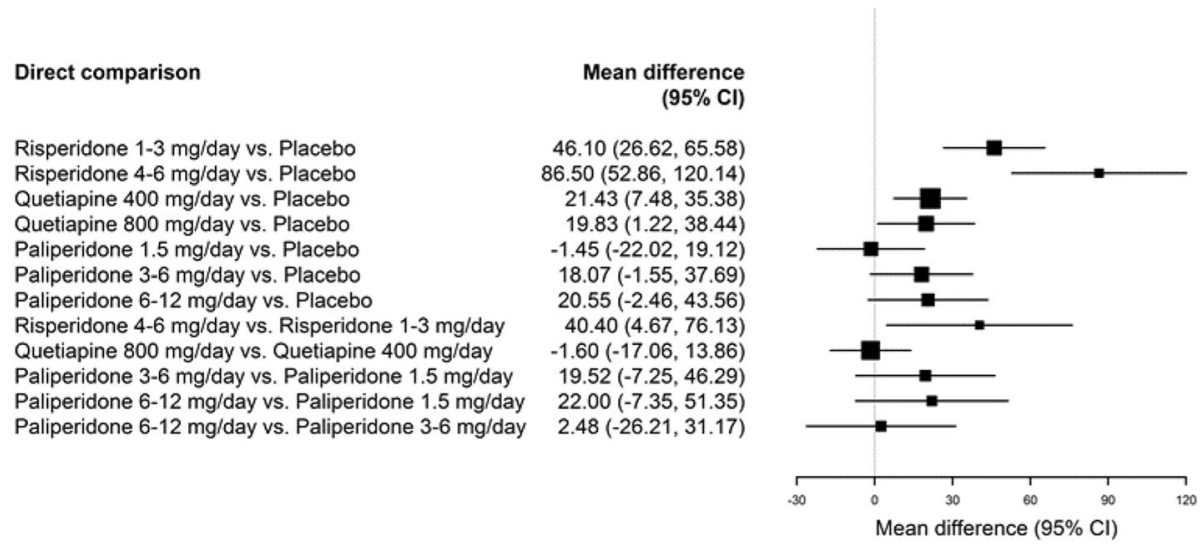

Fig. 4 Forest plot of mean change from baseline for SGAs presented in RCTs, female population only

baseline prolactin levels for males and females were available separately and differed between sexes [29, 31]. Pandina et al. (2012) reported baseline prolactin levels for males of $18.6 \mathrm{ng} / \mathrm{mL}, 40.4 \mathrm{ng} / \mathrm{mL}$, and $55.8 \mathrm{ng} / \mathrm{mL}$ and baseline prolactin levels for females of $14.3 \mathrm{ng} / \mathrm{mL}$, $73.6 \mathrm{ng} / \mathrm{mL}$, and $100.3 \mathrm{ng} / \mathrm{mL}$ for the treatment-naïve, treatment-experienced, and open-label arms, respectively [29]. This difference was not as marked, but still different, in the paper by Schimmelmann and colleagues (2007), which reported levels of $12.6 \mathrm{ng} / \mathrm{mL}$ and $22.9 \mathrm{ng} / \mathrm{mL}$ for males and females, respectively [31].

\section{Outcomes}

Outcomes were typically reported after 12 or 24 weeks of treatment. Prolactin-related adverse events were reported in three studies; two reporting on patient experience with varying levels of risperidone $[29,30]$ and one with quetiapine $200-800 \mathrm{mg}$ daily [31]. Pandina et al. (2012) reported 36 (9 \%) prolactin-related adverse events, 14 cases (3.6\%) of gynecomastia/galactorrhea, 3 cases $(1.0 \%)$ of amenorrhea/dysmenorrhea, and a single case $(<0.5 \%)$ of impotence or decreased libido across study arms. These adverse events were not reported by treatment arm, which differed with regards to prior treatment experience and treatment duration. In a study of patients treated biweekly with $25-50 \mathrm{mg}$ of risperidone for 24 weeks, 5 cases (16\%) of prolactin-related adverse events, comprising 2 cases $(6.5 \%)$ of gynecomastia/galactorrhea and 3 cases $(9.7 \%)$ of amenorrhea/dysmenorrhea, were reported [30]. A single study assessing quetiapine reported 3 cases $(5.4 \%)$ of gynecomastia/galactorrhea, 4 cases $(7.1 \%)$ of amenorrhea/dysmenorrhea, and 9 cases (16.1\%) of impotence or decreased libido [31].

Mean change from baseline in prolactin levels is presented in Table 4. Mean changes were available in four studies; one reporting on quetiapine [31] and three on risperidone $[27,29,30]$. Patients treated with quetiapine $200-800 \mathrm{mg} /$ day experienced a mean decrease of $1.4 \mathrm{ng} / \mathrm{mL}$ (standard deviation [SD] 21.1) [31]. Changes in patients treated with risperidone varied considerably, from a mean increase of $66.8 \mathrm{ng} / \mathrm{mL}$ (SD 41.8) to a mean decrease of $13.1 \mathrm{ng} / \mathrm{mL}$ (SD 17.1).

Change from baseline stratified by sex was available in two studies $[29,31]$. In the first study, male patients treated with quetiapine $200-800 \mathrm{mg} /$ day were found to have a mean decrease of $4.5 \mathrm{ng} / \mathrm{mL}$ (SD 5.7), while females experienced an increase of $3.2 \mathrm{ng} / \mathrm{mL}$ (SD 21.7) [31]. In the second study, male patients treated with risperidone $2-6 \mathrm{mg} /$ day who were previously treatment-naïve were found to have a mean increase of $29.1 \mathrm{ng} / \mathrm{mL}$ (SD 32.6), whereas treatment-experienced patients were found to decrease levels by $6.2 \mathrm{ng} / \mathrm{mL}$ (SD 22.2). Female patients experienced larger changes from baseline, with treatment-naïve patients reporting mean increases of $83.4 \mathrm{ng} / \mathrm{mL}$ (SD 44.7) and treatment-experienced patients reporting a mean decrease of $14.0 \mathrm{ng} / \mathrm{mL}$ (49.3). In the open-label arm of this risperidone study, which consisted of patients with varying levels of treatment experience, males were reported to have a mean increase of $3.7 \mathrm{ng} / \mathrm{mL}$ (SD 28.5) while females reported a mean increase of $11.5 \mathrm{ng} / \mathrm{mL}$ (SD 43.3) [29].

\section{Risk of bias assessment}

The Cochrane risk of bias tool was used to assess the quality of included RCTs [18]. A lack of reporting on sequence generation [21-24] and allocation concealment $[22-24,26]$, as well as a lack of completeness for primary outcome data [22] were raised as causes for concern in a number of trials. Other sources of bias, which included lack of reporting on specific baseline characteristics (prolactin levels) and prior treatment experience, were deemed high in three studies [22-24]. Overall, included RCTs were deemed to have a minimal risk of bias. Results of this evaluation are available in the Additional file 1: Figure S1 and Additional file 2: Table S1. 
The NOS was used to assess the quality of included observational studies [19]. All studies, with the exception of Ruan et al. 2010, studied populations of patients whose outcomes may be generalized to all pediatric patients with schizophrenia being treated with antipsychotics. Ruan et al. 2010 combined two study populations, one previously treated with olanzapine and the other previously treated with oral risperidone. However, baseline prolactin levels in these two groups were differed greatly (31.3 ng/mL [SD 11.3] and 87.4 $\mathrm{ng} / \mathrm{mL}$ [SD 15.5] for patients with previous olanzapine and risperidone experience, respectively). As prolactin levels are measured biochemically, ascertainment of exposure presents minimal risk of bias. The included observational studies were considered comparable in that diagnoses and patient ages included were similar. However, differences in exclusion criteria for baseline disease severity and study durations did not allow for robust comparisons (one study had a follow-up time of less than four weeks, shorter than any of the included RCTs [27]). In all studies, loss-to-follow up was accounted for. Overall, this observational evidence presents a medium to high risk of bias.

\section{Discussion}

This review was designed to evaluate the frequency of prolactin-related adverse events and to consolidate/ quantify differences in prolactin level changes between antipsychotics in pediatric patients with schizophrenia and schizophrenia spectrum disorders. No definitive conclusions can be drawn regarding differences in prolactin-related adverse events between antipsychotics due to the paucity of data. However, analyses of mean change from baseline in prolactin levels demonstrated some differences between treatments.

A review of the evidence reported in RCTs found that, in analyses of both the overall population and the population stratified by patient sex, risperidone at 1-3 $\mathrm{mg} /$ day and 4-6 mg/day was associated with an increase in prolactin levels compared to baseline. This change was particularly marked in females. When compared to placebo, both doses of risperidone produced greater increases in prolactin levels. Risperidone 4-6 mg/day was found to produce greater increases than risperidone $1-3 \mathrm{mg} /$ day in the overall population. However, this trend was not observed in males. A single-arm observational study by Duval et al. reported outcomes in patients treated with risperidone 1-6 mg/day. They found that prolactin levels increased, on average, in these patients after three weeks of treatment. This finding is in line with the findings in the RCT evidence. A three-arm study by Pandina et al. in patients treated with risperidone 2$6 \mathrm{mg} /$ day reported outcomes at 24 weeks for two arms, one treatment-naïve and the other treatmentexperienced, and 48 weeks for the remaining openlabel arm. Patients in the treatment-experienced and open-label arms showed relatively little change in prolactin levels. However, this may be attributed to previous treatment experience. Patients in the treatmentnaïve arm experienced increases in prolactin levels, with a more marked increase in females. However, these changes were accompanied by large dispersions. The findings in the treatment-naive patients reflect those of the RCT evidence that risperidone is associated with an overall increase in prolactin levels. Differences in patient characteristics such as treatment-experience, which was not reported in the included RCTs, and time horizons may explain the contradictory nature between the observational and RCT evidence.

Paliperidone at doses of 3-6 mg/day and 6-12 mg/day, but not $1.5 \mathrm{mg} /$ day, was associated with increases in prolactin levels between baseline and endpoint and when compared to placebo. When evaluating the evidence stratified by patient sex, the same trend was observed in males but not in females. Comparisons in the overall population between paliperidone doses suggest that paliperidone 3-6 $\mathrm{mg} /$ day and $6-12 \mathrm{mg} /$ day both increase prolactin more than the $1.5 \mathrm{mg} /$ day dose. However, this trend only held for males when results were stratified by patient sex. The difference in change in prolactin between paliperidone $6-12 \mathrm{mg} /$ day and 3-6 mg/day was not statistically significant. No observational study was identified reporting on patients treated with paliperidone.

Changes in prolactin levels were significant for both doses of quetiapine and for aripiprazole in the RCT evidence, with mean decreases compared to baseline. When compared to placebo, quetiapine $800 \mathrm{mg} /$ day reported an increase from baseline in prolactin levels over the study period. This change was statistically significant for females, but not for males. In the single observational study reporting on changes in patients treated with quetiapine, a decrease in prolactin levels in the overall and male only populations was reported, with a reported increase for the female population. It is of note that the dispersions reported with these values were wide. However, direct comparisons across these bodies of evidence must be made with caution, given that there a number of factors that may contribute in explaining the disparity of these results. For example, the doses included in the observational study varied considerably, from $200 \mathrm{mg} /$ day to $800 \mathrm{mg} /$ day, while patients in the RCT were treated with doses of $400 \mathrm{mg} /$ day and $800 \mathrm{mg} /$ day. Moreover, the observational study reported outcomes at 12 weeks, while the RCT reported outcomes at 6 weeks.

The findings of this review are generally in agreement with recent reviews on changes in prolactin levels 
relating to the use of antipsychotic medications [33, 34]. A 2012 review by Cohen et al. reported on short-term (3 to 12 week) outcomes from SGA use in child and adolescent patients for the management of a number of diagnoses, including schizophrenia, bipolar disorder, and conduct disorder. They identified four studies on the use of aripiprazole where patients were reported to have statistically significant decreases in prolactin levels compared to baseline. This is consistent with the findings of the current review. However, when changes from baseline were compared between aripiprazole and placebo in the current review, this decrease was not found to be statistically significant. Compared to placebo, Cohen reported that risperidone and olanzapine both produced increases in prolactin levels, which is consistent with the findings of the current review. Similarly, Leucht et al. reported increases in prolactin levels associated with the use of risperidone and paliperidone. However, the dose of paliperidone was not specified. In the current review, paliperidone was found to increase prolactin levels at the 3-6 $\mathrm{mg} /$ day and 6-12 $\mathrm{mg} /$ day dose ranges, but not in patients treated with the $1.5 \mathrm{mg} /$ day regimen. Consistent with the current review, treatment with aripiprazole was not found to increase prolactin compared to placebo. Leucht et al. also found that quetiapine was not associated with increases when compared to placebo. In the current review, however, treatment with quetiapine at both the $400 \mathrm{mg} /$ day and $800 \mathrm{mg} /$ day doses was found to increase prolactin levels relative to placebo in the overall population. Treatment with olanzapine was found, by Leucht et al., to increase prolactin relative to placebo, which is consistent with the current review. While the review by Leucht et al. was also in patients with schizophrenia, the scope was not limited to children. This difference may explain discrepancies between findings.

Differences between male and female patients with respect to the prolactin elevation response, particularly the more pronounced responses among female patients, have been described in the literature. A 1992 study of schizophrenia patients found that serum prolactin increased more markedly in female patients following the commencement of neuroleptic medications, despite the female patients receiving lower doses of treatment [35]. Interestingly, the individual patient variability in response was also higher in females than males. These findings supported an earlier study also conducted in patients with schizophrenia [36]. Similar trends were observed in the current review, with females having a higher prolactin elevation response compared to males when treated with risperidone and quetiapine. However, an opposite trend was observed for patients treated with paliperidone 3-6 mg/day or 6-12 mg/day.

Typically, reference ranges for biochemical markers are established to inform physicians on levels at which these markers may pose a risk to patient safety. While the endocrinology literature documents the effect of prolactin on the body, no acceptable ranges have been established for serum prolactin levels in the pediatric population. A 1973 study of 19 normal children aged 2-12 years and 54 adolescent children aged 13-16 years estimated mean prolactin levels in both male and females to be approximately $5 \mathrm{ng} / \mathrm{mL}$ [37]. However, patient characteristics were poorly described, so generalizations must be made with caution. A study by Cook et al. proposed reference ranges (2.5 to 97.5 percentiles) for pediatric prolactin based on a hospital sample [38]. Based on this patient sample, the normal ranges for males and females aged 13 to 15 years of age were defined as $1.6-16.6 \mathrm{ng} / \mathrm{mL}$ and $3.0-14.4 \mathrm{ng} / \mathrm{mL}$, respectively. However, the level of elevation in prolactin levels that warrants concern for adverse events in children has yet to be defined. According to some clinical investigators, however, prolactin levels between $18 \mathrm{ng} / \mathrm{mL}$ and $30 \mathrm{ng} / \mathrm{mL}$ are rarely associated with adverse events related to the hypothalamic-pituitary-gonadal axis, though prolonged elevations nearing $100 \mathrm{ng} / \mathrm{mL}$ necessitate clinical investigation [13]. It is, therefore, difficult to determine what prolactin levels substantially increase the risk of prolactin-related adverse events in these patients [39]. Therefore, although some trends emerged in the analysis of prolactin changes in patients treated with second-generation antipsychotics, the lack of causal association precludes conclusions being drawn between the use of any particular antipsychotic and prolactin-related adverse events.

This review is useful in that the process and design was well structured, with screening and data extraction conducted using a duplicate, consensus-based approach. Additionally, the body of evidence considered was broad, including both RCTs and observational studies. While observational studies may lack the methodological rigor of RCTs, they may still be considered an important source of information on treatment efficacy and safety. Despite this, this review presents some limitations. First, few trials were identified from the available literature and many were characterized by small sample sizes. Secondly, the observational studies suffered from significant heterogeneity in study populations, both in comparison to one another and to the RCT evidence base. Similarly, the patients represented in the evidence base were primarily Caucasians, which may limit the generalizability of the findings to the wider patient population. Followup time, prior treatment experience, and patient diagnoses also differed significantly in the observational studies. Thirdly, estimates of dispersion were large in each of the RCTs. Finally, while the mechanism is clear between prolactin and breast development/lactation, there is no conclusive research to confirm this association epidemiologically. In a similar vein, it was not possible, from the 
included studies, to directly associate changes in prolactin levels with patient adverse events. While this review was focused on pediatric patients with schizophrenia, in order to present outcomes for a well-defined clinical area, it must also be recognized that the potential for treatment-related prolactin elevation is not limited to this population. The risk and effects of prolactin elevation are an important clinical concern in other diagnostic areas and should be explored.

\section{Conclusion}

In summary, while some trends in prolactin level changes from baseline emerged in the data, no definitive conclusions on the association between particular antipsychotic use and prolactin-related adverse events can be drawn. Nevertheless, clinician monitoring of prolactin-related adverse events is pertinent. Future trials examining the effects of antipsychotic medications for pediatric schizophrenia and schizophrenia spectrum disorder patients should emphasize reporting on safety outcomes, with studies of adequate duration to allow for the observation of these outcomes. Routine monitoring of serum prolactin levels in study participants is encouraged, as this may allow investigators to directly attribute adverse events to changes in prolactin.

\section{Additional files}

Additional file 1: Figure S1. Evaluation of RCTs with the Cochrane risk of bias assessment. (PDF $267 \mathrm{~kb}$ )

Additional file 2: Table S1. Evaluation of included observational studies with the Newcastle-Ottawa Scale. (DOCX 25 kb)

\section{Abbreviations \\ BPRS: Brief psychiatric rating scale; CGAS: Children's global assessment scale; CGI-S: Clinical global impressions scale; DSM-IV: Diagnostic and statistical manual of mental disorders, 4th edition; FGA: First-generation antipsychotic; ITT: Intent-to-treat; K-SADS-PL: Kiddie schedule for affective disorders and schizophrenia for school-age children, present and lifetime version; NOS: Newcastle-Ottawa scale; NR: Not reported; PANSS: Positive and negative syndrome scale; RCT: Randomized controlled trial; SD: Standard deviation; SGA: Second-generation antipsychotic.}

\section{Acknowledgements}

Not applicable.

\section{Funding}

This work was funded by a Canadian Institutes of Health Research (CIHR) Drug Safety and Effectiveness (DSEN) grant.

\section{Availability of data and materials}

Not applicable.

\section{Authors' contributions}

The study protocol was developed by ED, SK, KR, EJM, and KTh. EM and KTh provided scientific oversight. SK and KR provided clinical guidance. Literature screening and data collection was conducted by ED, MJZ, KTo, and PW. Statistical analyses were conducted by KTo and KTh. The first draft was written by ED, MJZ, and KTo. All authors read, revised, and approved the final manuscript.

\section{Competing interests}

The authors declare that they have no competing interests.

\section{Consent for publication}

Not applicable.

Ethics approval and consent to participate

Not applicable.

\section{Author details}

${ }^{1}$ Faculty of Health Sciences, University of Ottawa, Ottawa, ON, Canada. ${ }^{2}$ Department of Clinical Epidemiology and Biostatistics, McMaster University, Hamilton, ON, Canada. ${ }^{3}$ Department of Pharmacy of the Ottawa Hospital, Ottawa Hospital Research Institute, Ottawa, ON, Canada. ${ }^{4}$ Department of Psychiatry, Ottawa Hospital, Ottawa, ON, Canada.

Received: 12 January 2016 Accepted: 13 October 2016

Published online: 09 November 2016

\section{References}

1. A roadmap to key pharmacologic principles in using antipsychotics. Prim Care Companion J Clin psychiatry 2007, 9(6):444-454.

2. McClellan J, Werry J. Practice parameters for the assessment and treatment of children and adolescents with schizophrenia. American Academy of Child and Adolescent Psychiatry. J Am Acad Child Adolesc Psychiatry. 1994;33(5):616-35.

3. Pringsheim T, Lam D, Patten SB. The pharmacoepidemiology of antipsychotic medications for Canadian children and adolescents: 2005-2009. J Child Adolesc Psychopharmacol. 2011;21(6):537-43.

4. Correll CU. Antipsychotic use in children and adolescents: minimizing adverse effects to maximize outcomes. J Am Acad Child Adolesc Psychiatry. 2008;47(1):9-20.

5. Vitiello B, Correll C, van Zwieten-Boot B, Zuddas A, Parellada M, Arango C. Antipsychotics in children and adolescents: increasing use, evidence for efficacy and safety concerns. Eur Neuropsychopharmacol. 2009;19(9):629-35.

6. Alessi-Severini S, Biscontri RG, Collins DM, Sareen J, Enns MW. Ten years of antipsychotic prescribing to children: a Canadian population-based study. Can J Psychiatry. 2012;57(1):52-8.

7. Kealey E, Scholle SH, Byron SC, Hoagwood K, Leckman-Westin E, Kelleher K, Finnerty M. Quality concerns in antipsychotic prescribing for youth: a review of treatment guidelines. Acad Pediatr. 2014;14(5 Suppl):S68-75.

8. Bostwick JR, Guthrie SK, Ellingrod VL. Antipsychotic-induced hyperprolactinemia. Pharmacotherapy. 2009;29(1):64-73.

9. Ho J, Panagiotopoulos C, McCrindle B, Grisaru S, Pringsheim T. Management recommendations for metabolic complications associated with second generation antipsychotic use in children and youth. J Can Acad Child Adolesc Psychiatry. 2011;20(3):234-41.

10. Knegtering $H$, van der Moolen $A E$, Castelein $\mathrm{S}$, Kluiter $H$, van den Bosch $R$ J. What are the effects of antipsychotics on sexual dysfunctions and endocrine functioning? Psychoneuroendocrinology. 2003;28 Suppl 2:109-23.

11. Byerly M, Suppes T, Tran QV, Baker RA. Clinical implications of antipsychoticinduced hyperprolactinemia in patients with schizophrenia spectrum or bipolar spectrum disorders: recent developments and current perspectives. J Clin Psychopharmacol. 2007;27(6):639-61.

12. Anderson GM, Scahill L, McCracken JT, McDougle CJ, Aman MG, Tierney E, Arnold LE, Martin A, Katsovich L, Posey DJ, et al. Effects of short- and longterm risperidone treatment on prolactin levels in children with autism. Biol Psychiatry. 2007;61(4):545-50.

13. Findling RL, Kusumakar V, Daneman D, Moshang T, De Smedt G, Binder C. Prolactin levels during long-term risperidone treatment in children and adolescents. J Clin Psychiatry. 2003;64(11):1362-9.

14. Halbreich U, Kinon BJ, Gilmore JA, Kahn LS. Elevated prolactin levels in patients with schizophrenia: mechanisms and related adverse effects. Psychoneuroendocrinology. 2003;28 Suppl 1:53-67.

15. Montgomery J, Winterbottom E, Jessani M, Kohegyi E, Fulmer J, Seamonds B, Josiassen RC. Prevalence of hyperprolactinemia in schizophrenia: association with typical and atypical antipsychotic treatment. J Clin Psychiatry. 2004;65(11):1491-8.

16. Pfeiffer D, Brule-Brown D. Second-generation antipsychotics and cardiometabolic adverse reactions in children and adolescents. Can Adverse React Newsl. 2012;22(1):1-2. 
17. Druyts E, Eapen S, Wu P, Thorlund K. The risk of elevated prolactin levels in pediatric patients exposed to antipsychotics for the treatment of schizophrenia and schizophrenia spectrum disorders: protocol for a systematic review and meta-analysis. Syst Rev. 2014;3:116.

18. Higgins JP, Altman DG, Gotzsche PC, Juni P, Moher D, Oxman AD, Savovic J, Schulz KF, Weeks L, Sterne JA. The Cochrane Collaboration's tool for assessing risk of bias in randomised trials. BMJ. 2011;343:d5928.

19. Cota GF, de Sousa MR, Fereguetti TO, Rabello A. Efficacy of anti-leishmania therapy in visceral leishmaniasis among HIV infected patients: a systematic review with indirect comparison. PLoS Negl Trop Dis. 2013;7(5):e2195.

20. Miller JJ. The Inverse of the Freeman-Tukey Double Arcsine Transformation. Am Stat. 1978;32(4):138.

21. Findling RL, McKenna K, Earley WR, Stankowski J, Pathak S. Efficacy and safety of quetiapine in adolescents with schizophrenia investigated in a 6-week, double-blind, placebo-controlled trial. J Child Adolesc Psychopharmacol. 2012;22(5):327-42.

22. Findling RL, Robb A, Nyilas $M$, Forbes RA, Jin N, Ivanova $S$, Marcus $R$, McQuade RD, Iwamoto T, Carson WH. A multiple-center, randomized, double-blind, placebo-controlled study of oral aripiprazole for treatment of adolescents with schizophrenia. Am J Psychiatr. 2008;165(11):1432-41.

23. Haas M, Unis AS, Armenteros J, Copenhaver MD, Quiroz JA, Kushner SF. A 6-week, randomized, double-blind, placebo-controlled study of the efficacy and safety of risperidone in adolescents with schizophrenia. J Child Adolesc Psychopharmacol. 2009;19(6):611-21.

24. Kryzhanovskaya L, Schulz SC, McDougle C, Frazier J, Dittmann R, RobertsonPlouch C, Bauer T, Xu W, Wang W, Carlson J, et al. Olanzapine versus placebo in adolescents with schizophrenia: a 6-week, randomized, doubleblind, placebo-controlled trial. J Am Acad Child Adolesc Psychiatry. 2009;48:60-70.

25. Singh J, Robb A, Vijapurkar U, Nuamah I, Hough D. A randomized, doubleblind study of paliperidone extended-release in treatment of acute schizophrenia in adolescents. Biol Psychiatry. 2011;70(12):1179-87.

26. Haas M, Eerdekens M, Kushner S, Singer J, Augustyns I, Quiroz J, Pandina G, Kusumakar V. Efficacy, safety and tolerability of two dosing regimens in adolescent schizophrenia: double-blind study. $\mathrm{Br} J$ Psychiatry. 2009;194:158-64.

27. Duval F, Guillon MS, Mokrani MC, Crocq MA, Garcia Duarte F. Relationship between prolactin secretion, and plasma risperidone and 9hydroxyrisperidone concentrations in adolescents with schizophreniform disorder. Psychoneuroendocrinology. 2008;33(2):255-9.

28. Kumra S, Kranzler H, Gerbino-Rosen G, Kester HM, DeThomas C, Cullen K, Regan J, Kane JM. Clozapine versus "high-dose" olanzapine in refractory early-onset schizophrenia: An open-label extension study. J Child Adolesc Psychopharmacol. 2008;18(4):307-16.

29. Pandina G, Kushner S, Karcher K, Haas M. An open-label, multicenter evaluation of the long-term safety and efficacy of risperidone in adolescents with schizophrenia. Child Adolesc Psychiatry Ment Health. 2012;6:ArtID 23.

30. Ruan L, Hu S, Huang M, Hu J, Cai W. Efficacy and safety of long-acting risperidone on early onset schizophrenia in adolescent patients. Afr J Pharm Pharmacol. 2010;4(5):184-92.

31. Schimmelmann BG, Mehler-Wex C, Lambert M, Schulze-zur-Wiesch C, Koch E, Flechtner HH, Gierow B, Maier J, Meyer E, Schulte-Markwort M. A prospective 12-week study of quetiapine in adolescents with schizophrenia spectrum disorders. J Child Adolesc Psychopharmacol. 2007;17(6):768-78.

32. Kumra S, Kranzler H, Gerbino-Rosen G, Kester HM, De Thomas C, Kafantaris V, Correll CU, Kane JM. Clozapine and "high-dose" olanzapine in refractory early-onset schizophrenia: a 12-week randomized and double-blind comparison. Biol Psychiatry. 2008;63(5):524-9.

33. Cohen D, Bonnot O, Bodeau N, Consoli A, Laurent C. Adverse effects of second-generation antipsychotics in children and adolescents: a Bayesian meta-analysis. J Clin Psychopharmacol. 2012;32(3):309-16.

34. Leucht S, Cipriani A, Spineli L, Mavridis D, Orey D, Richter F, Samara M, Barbui C, Engel RR, Geddes JR, et al. Comparative efficacy and tolerability of 15 antipsychotic drugs in schizophrenia: a multiple-treatments metaanalysis. Lancet. 2013;382(9896):951-62.

35. Kuruvilla A, Peedicayil J, Srikrishna G, Kuruvilla K, Kanagasabapathy AS. A study of serum prolactin levels in schizophrenia: comparison of males and females. Clin Exp Pharmacol Physiol. 1992;19(9):603-6.

36. Meltzer HY, Busch DA, Fang VS. Serum neuroleptic and prolactin levels in schizophrenic patients and clinical response. Psychiatry Res. 1983;9(4):271-83.
37. Guyda HJ, Friesen HG. Serum prolactin levels in humans from birth to adult life. Pediatr Res. 1973;7(5):534-40.

38. Cook JF, Hicks JM, Godwin ID, Bailey J, Soldin SJ. Pediatric reference ranges for prolactin. Clin Chem. 1992;38(6):959.

39. Pappagallo M, Silva R. The effect of atypical antipsychotic agents on prolactin levels in children and adolescents. J Child Adolesc Psychopharmacol. 2004;14(3):359-71.

\section{Submit your next manuscript to BioMed Central and we will help you at every step:}

- We accept pre-submission inquiries

- Our selector tool helps you to find the most relevant journal

- We provide round the clock customer support

- Convenient online submission

- Thorough peer review

- Inclusion in PubMed and all major indexing services

- Maximum visibility for your research

Submit your manuscript at www.biomedcentral.com/submit
) Biomed Central 